\title{
1 Study on a digital pulse processing algorithm based on template-matching for high-
}

2

3

4

5

6

7

8

9

10

11

12

13

14

15

16

17

18

19

20

\section{1. Introduction}

22 Innovative systems with increased sensitivity and resolution are in great demand to detect diversion and to prevent

23 misuse in support of nuclear materials management for the U.S. fuel cycle [1-3]. Nuclear fission is the most

24 important multiplicative process involved in non-destructive active interrogation. Among others, unique delayed

25 gamma-ray spectra exist for fissionable isotopes and can be used for isotopic composition measurement [4-16]. A

26 major challenge in utilizing delayed fission gamma rays emitted after active interrogation for safeguards

27 applications is to perform high-resolution spectroscopy measurements at an ultra-high throughput rate [17]. In our 

could be re-written as: $\left[\begin{array}{c}y_{1} \\ \vdots \\ y_{D}\end{array}\right]=\left[\begin{array}{ccc}t_{11} & \cdots & t_{1 B} \\ \vdots & \ddots & \vdots \\ t_{D 1} & \cdots & t_{D B}\end{array}\right]\left[\begin{array}{c}s_{1} \\ \vdots \\ s_{B}\end{array}\right]$. Here, $\mathrm{s}_{\mathrm{j}}(\mathrm{j}=1, \ldots, \mathrm{B})$ is a vector containing the amplitude of the recent experimental study at Idaho Accelerator Center, the high-energy x-ray beam with maximum energy of 22 $\mathrm{MeV}$ generated by a pulsed linac was used as the photon source. Delayed $\gamma$-rays emitted from photon-induced fission reactions inside the samples were measured between adjacent linac pulses with several gamma spectroscopy systems. However, these systems could not work within several milliseconds following each linac pulse although they were well shielded to get rid of irradiation directly from the interrogation source [10]. In addition to modification of front-end electronics to allow rapid recovery from saturation after each linac pulse, innovative pulse analysis algorithms are needed to achieve high-throughput. Using traditional pulse processing algorithms, throughput rate could be significantly impacted when the input rate is high, due to pile-up rejection. Recently, pulse pile-up recovery based on template-matching has been proved to be an effective approach to achieve high throughput gamma spectroscopy [18-21]. The work presented here is primarily based upon the methodology developed by Scoullar and Evans [18-19]. A detailed discussion of our algorithm is given below, followed by implementations on signals from a NaI detector, a silicon drift detector and a HPGe detector. Compared with traditional trapezoidal shaping, a much higher throughput rate could be achieved using the template-matching technique. This would be very beneficial in high-rate counting scenarios such as pulsed linac based active interrogations.

\section{Discussion of the algorithm}

In a simplified model, the output signal $y(t)$ from a gamma-ray detector is the convolution of the incident gamma ray signal $s(t)$ with the detector response matrix $M: y(t)=s(t) * M$. Thus, if the response matrix can be accurately determined, an estimation of the incident signal can be obtained through a deconvolution process. The incident signal is normally modeled as a train of delta functions, with random time of arrival and amplitude. The detector response is considered to be time-invariant and can be pre-determined. The first step of the algorithm discussed here is to determine the time of arrival for each pulse by the use of a narrow trapezoidal filter [22]. This can greatly reduce the complexity of the problem. Once the time of arrival for each pulse is determined, the detection process 
54 point. Assuming the time-invariant impulse response of the detector was known (i.e. the template), the matrix $\mathrm{t}_{\mathrm{ij}}$ can 55 then be calculated once the time of arrival for each pulse is determined. Each row of the matrix would just be the 56 impulse response with various amount of delay. Ideally, since $y_{i}$ is measured and $t_{i j}$ can be calculated, one could 57 invert the response matrix and mathematically solve for the amplitude of the incident pulses. In fact, this was carried 58 out in our study and gave reasonably good results. However, there are some limitations that might push one away 59 from this direct approach. For example, if the dimension of the response matrix $t_{\mathrm{ij}}$ is too large (e.g. emission 60 imaging), the calculation involved in direct inversion is prohibitive. In addition, the response matrix might be sparse, 61 which also pose serious challenge to direct inversion. Furthermore, the measured signal $\mathrm{y}_{\mathrm{i}}$ could be noisy, 62 preventing an accurate numerical solution. Due to these factors, the response matrix is often nearly singular and the 63 equation above cannot be reliably solved for the amplitude of the incident pulses with direct inversion. In this case, 64 Maximum Likelihood Expectation Maximization (ML-EM) algorithm could be used to provide an estimation of the $65 \mathrm{~s}_{\mathrm{j}}$ vector using the well-known equation $s_{k}^{(n+1)}=s_{k}^{(n)}\left\{\frac{1}{\sum_{d=1}^{D} t_{d k}} \sum_{d=1}^{D}\left[\frac{y_{d}}{\sum_{b=1}^{B} t_{d b} x_{b}^{(n)}} t_{d k}\right]\right\}[23]$.

67 to be a double exponential function, simulating the anode signal from a PMT coupled with a scintillator detector.

68 The rising edge time constant is determined by the PMT response time (normally between 20 and 80 ns), while the 69 falling edge has the decay constant of the scintillator (e.g. $230 \mathrm{~ns}$ for NaI). The amplitude and the time of arrival of 70 each pulse were randomly generated. The simulated waveform was then generated as the superposition of these 71 individual pulses. In pulse processing, the shape (i.e. template) and the time of arrival of each pulse were assumed to 72 be known. Using the algorithm described above, the amplitude of each pulse can be calculated. As shown in Figure

731 , if the time of arrival and the template can be accurately determined, the result is very good even when a white 74 noise was added to the simulated anode signal. 


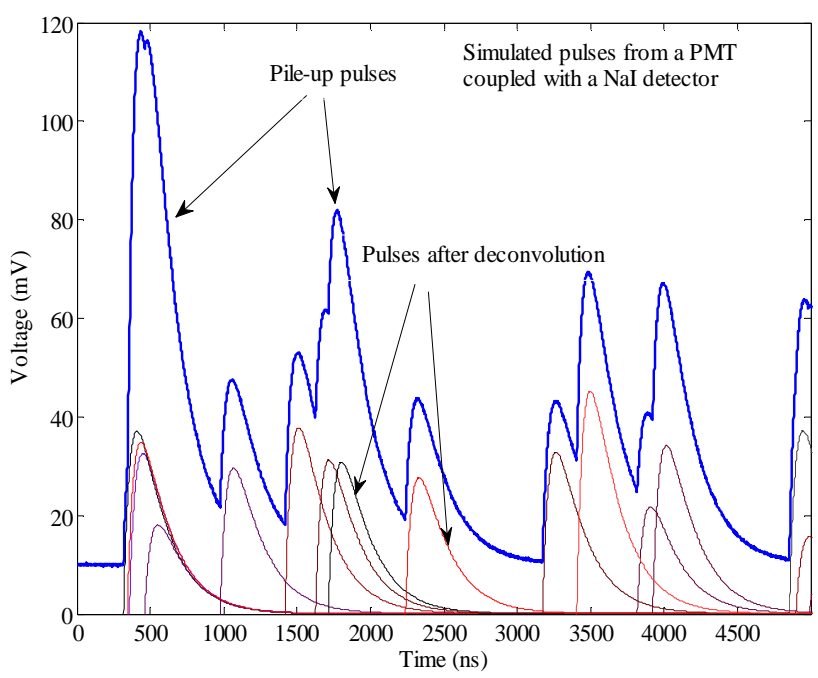

75

Fig. 1. The application of the template-matching algorithm to recover simulated piled-up pulses.

\section{Results and discussion}

The implementation of the template-matching algorithm on high rate NaI data, silicon drift detector data and HPGe data is discussed below. Its performance was also compared with a traditional pulse processing algorithm using trapezoidal filters.

\subsection{Implementation on signals from a NaI detector}

Firstly, the algorithm was implemented on signals obtained using a $\mathrm{NaI}$ detector. The detector used in these measurements was a Canberra Model 802 detector with a 2" by 2" crystal. The anode signal was directly digitized at a sampling rate of 100 MSPS using a National Instruments digitizer, model number PXIe-5122. A LabVIEW program was developed to enable streaming of digitized data onto a hard drive array at full speed for off-line processing. The digitized signal and the template are shown in Figure 2. Due to the large noise imposed on the anode signal, matching result was not ideal. Also, the determination of the time of arrival was challenging. Because of the fast rising edge, an offset of one or two points could cause a large deviation between the template and the measured data. In this case, the signal measured at very low count rate was used as the template for deconvolution. The performance of the template-matching algorithm was compared with traditional shaping methods. The shaping parameters used in the measurement with the Canberra Lynx system were $1 \mu$ s respectively for rise time and flat top time. At moderate count rate ( $200 \mathrm{kcps})$, the results were comparable, as shown in Figure 3. 
94

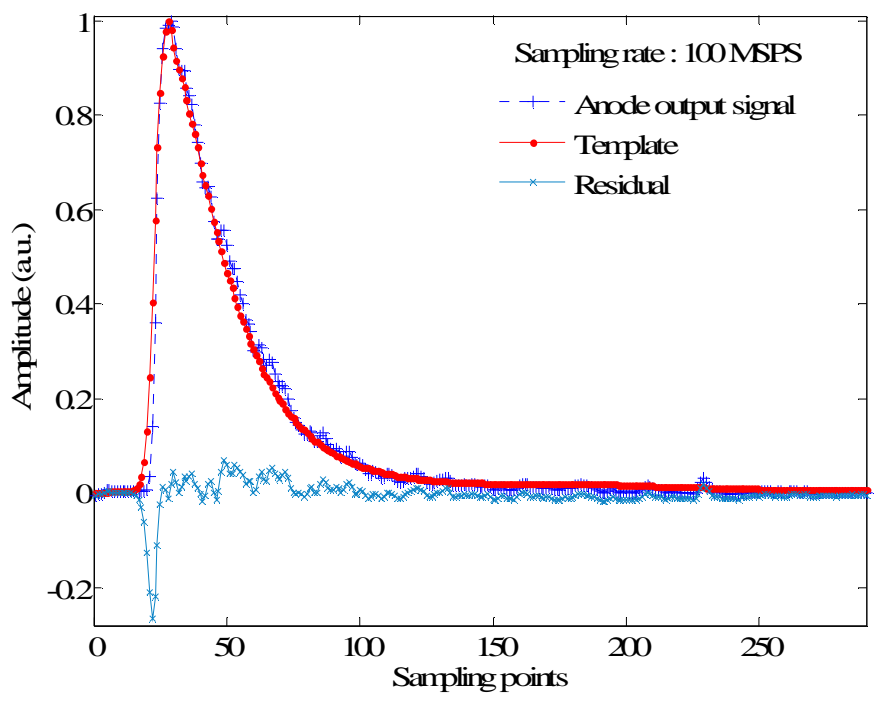

Fig. 2. The anode output signal, template and residual.

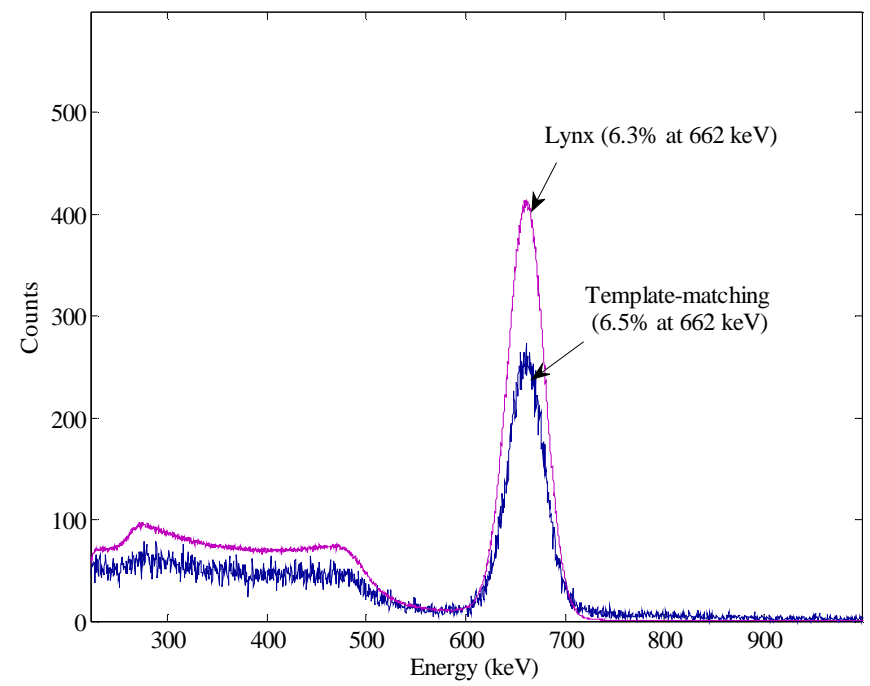

Fig. 3. Comparison between energy spectra measured with a Canberra Lynx system and reconstructed with the templatematching algorithm.

\subsection{Implementation on signals from a silicon drift detector}

The performance of the algorithm was also tested on high rate data measured with a silicon drift detector, provided by Southern Innovation. During the measurements, a Mn foil was irradiated with photons generated from an Amptek Mini-X tube to produce characteristic x-rays with energy of $5.89 \mathrm{keV}$ and $6.49 \mathrm{keV}$. The detector used was a Ketek $30 \mathrm{~mm}^{2}$ silicon drift detector [24]. Different input count rate (between $50 \mathrm{kcps}$ and $500 \mathrm{kcps}$ ) was 
105

106

107

108

109

110

111

112

113

114

115

116

117

118

119 produced by tuning the tube current. The $50 \mathrm{kcps}$ data was utilized to create a template by averaging over 200 pulses that did not suffer from pile-up. The energy resolution that the template-matching algorithm achieved at low input count rate (i.e. $50 \mathrm{kcps}$ ) was $131 \mathrm{eV}$ at $5.89 \mathrm{keV}$. Good Gaussian peak shape and reasonable energy resolution could still be obtained even at very high input rate (e.g. $500 \mathrm{kcps}$ ), as shown in Figure 4. To compare the performance of the template-matching algorithm with traditional pulse processing using trapezoidal filters, we first adjusted shaping parameters to achieve comparable energy resolution and compared throughput rate at each input rate. As shown in Figure 5 a much higher throughput rate (e.g. $467 \mathrm{kcps}$ vs $67 \mathrm{kcps}$ at $500 \mathrm{kcps}$ input rate) was achieved with the template-matching algorithm at very high input rate. The traditional pulse processing algorithm suffered huge decrease in throughput at high input rates due to pile-up rejection. The shaping parameters were then adjusted to achieve similar throughput rate using both algorithms at a certain input rate. Figure 6 shows the energy resolution as a function of input rate. As can be observed, using the template matching algorithm, resolution of less than $200 \mathrm{eV}$ at $5.89 \mathrm{keV}$ could be achieved even at $500 \mathrm{kcps}$. The resolution gradually degraded as input rate increased. This degradation is much more prominent for the traditional processing algorithm due to pile-up. In summary, Figures 46 clearly demonstrated the advantages of the template-match algorithm over traditional approaches: superior throughput with comparable energy resolution; superior energy resolution with comparable throughput.

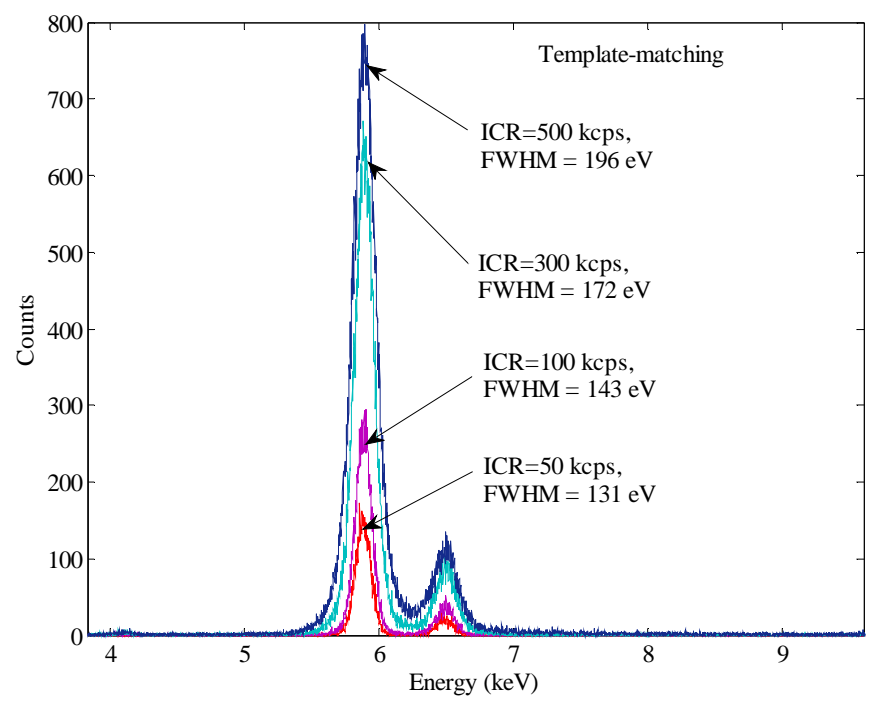




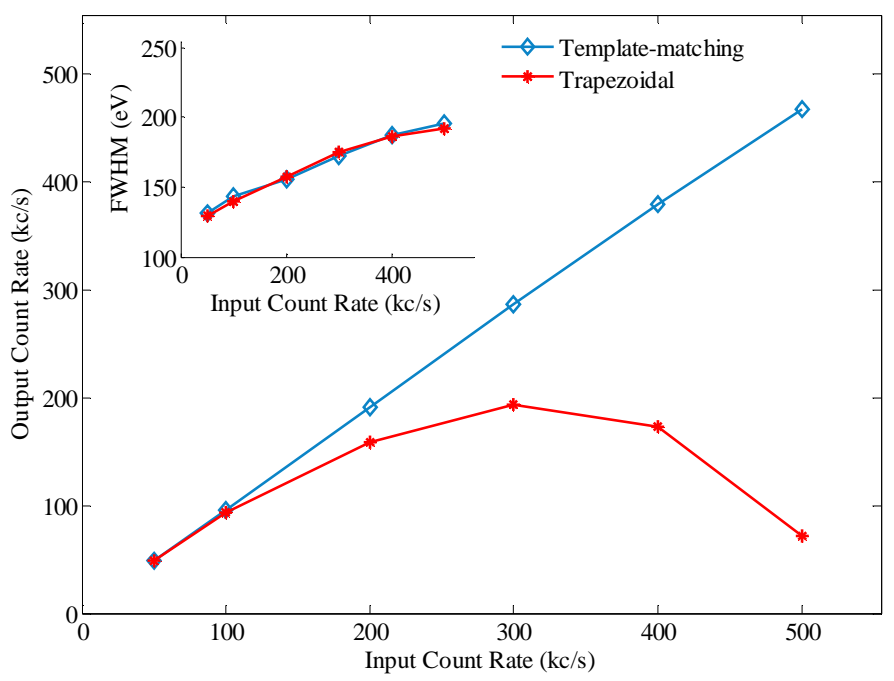

123 Fig. 5. Comparison between the template-matching algorithm and the trapezoidal filter (Output Count Rate vs Input Count Rate).

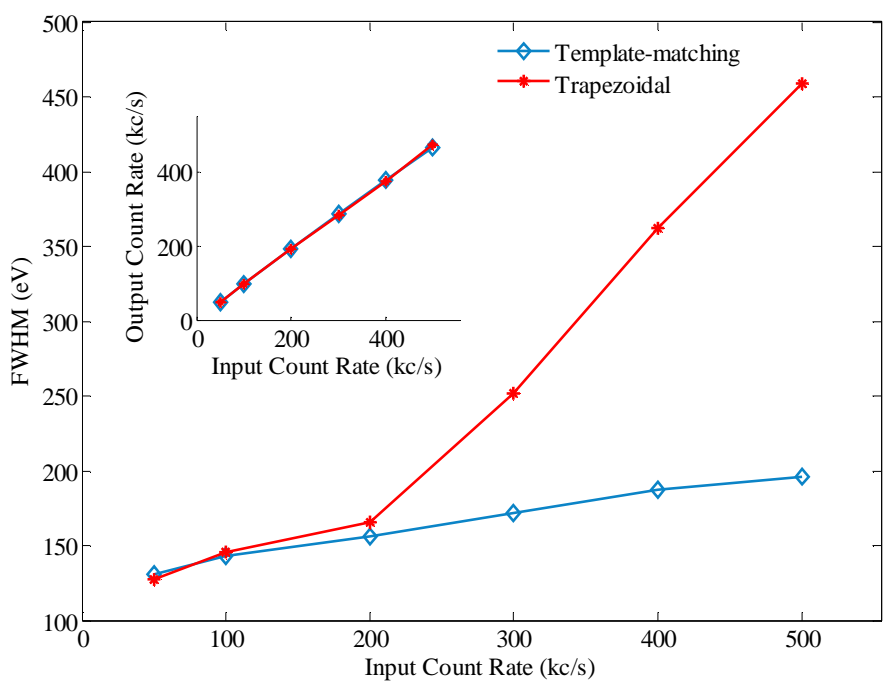

Fig. 6. Comparison between the template-matching algorithm and the trapezoidal filter (FWHM vs Input Count Rate).

\subsection{Implementation on signals from a HPGe detector}

128 The HPGe detector is the only practical choice that can provide excellent energy resolution in a wide energy range 129 as needed in measuring delayed gamma-ray spectra following active interrogation for nuclear safeguards applications. As discussed above, in such measurements, there are two issues that greatly limit the throughput of a HPGe detector: the long saturation after each linac pulse and the pulse pile-up due to high input rate. Our approaches to address this challenge include modification of the front-end electronics of a HPGe detector to allow 
133

134

135

136

137

138

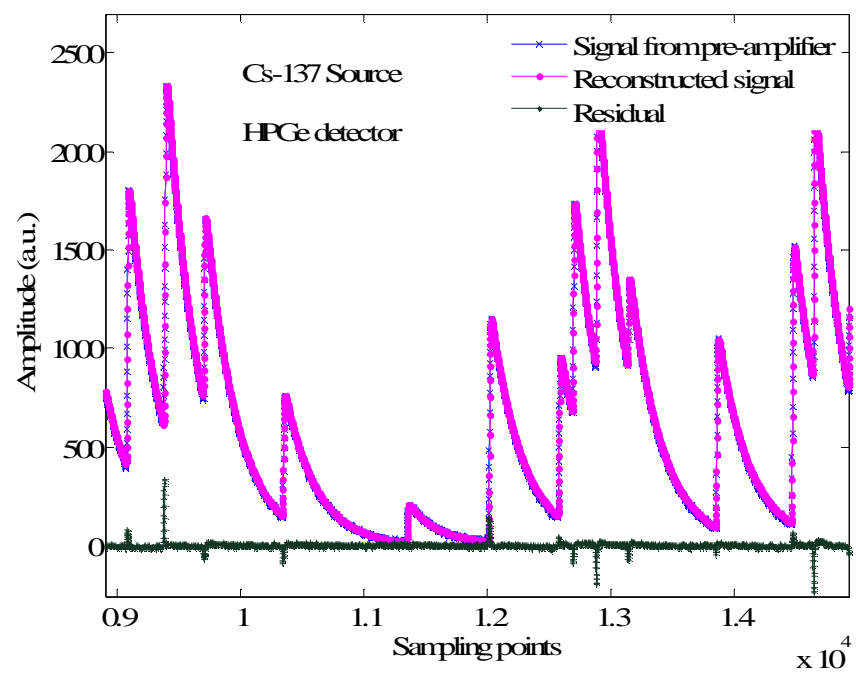

fast recovery from saturation due to large energy injection from the linac and the development of advanced digital signal processing techniques to improve the throughput rate with only small sacrifice in energy resolution. Here, we discuss the implementation of the template-matching algorithm on signals from a HPGe detector. It is quite challenging to implement the template-matching algorithm on HPGe signals because of the variation in pulse shape. To be specific, for a pulse from the pre-amplifier, the falling edge is determined by the RC constant of the preamplifier (e.g. $\sim 50 \mu \mathrm{s})$. On the other hand, the rising edge varies with the gamma-ray interaction position inside the detector. For simplicity, a generic template was used in current work. Pulses from the pre-amplifier were very well reconstructed at the falling edge, but a significant amount of deviation between the measured and reconstructed signals could be observed at the rising edge, as shown in Figure 7. In spite of this large deviation, good energy resolution at input rates up to $200 \mathrm{kcps}$ was achieved, as shown in Figure 8. A performance comparison between the template-matching algorithm and the traditional trapezoidal shaping was again performed and summarized in Table 1. Both the trapezoidal shaping and template-matching algorithms were implemented in customized scripts written in Matlab, running on a laptop (i5-2410M, 4.0 GB RAM, 64-bit Windows operating system). The raw digitized data was converted to mat file and then imported to Matlab. The sizes of the mat files for the $100 \mathrm{kcps}$ and $200 \mathrm{kcps}$ data were about $29 \mathrm{MB}$ and $33 \mathrm{MB}$, respectively. The processing time of the template-matching shaping algorithm was found to be much longer compared with that of the traditional trapezoidal filtering. The comparison results are shown in the last column of Table 1. The results showed the advantage of the template-matching approach despite the fact that a good template is difficult to construct.

Fig. 7. Verification of the reconstructed signal from pre-amplifier. 
153 154 155 156 157 158

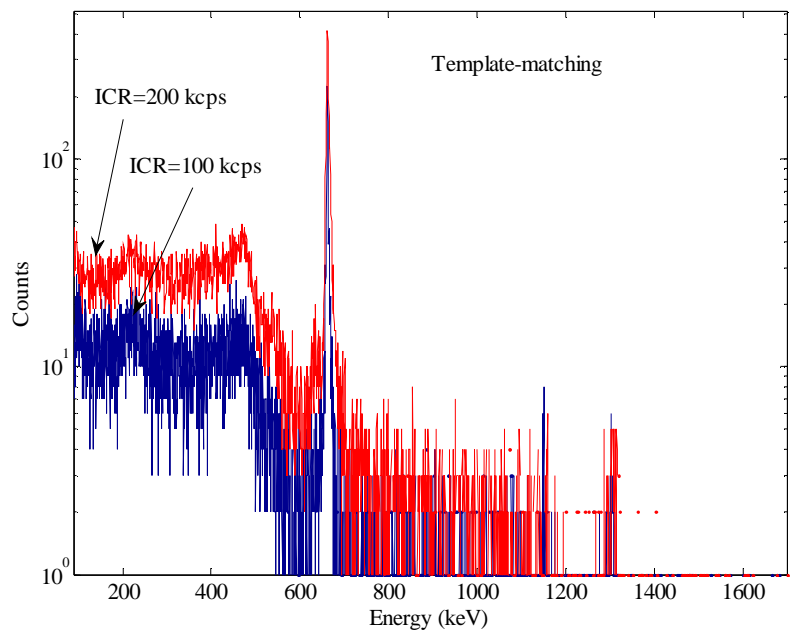

Fig. 8. Energy spectra reconstructed with the template-matching algorithm.

Table 1. Comparison between the template-matching algorithm and the trapezoidal filter (HPGe data).

\begin{tabular}{|c|c|c|c|c|}
\hline & $\begin{array}{c}\text { Input Count Rate } \\
(\mathrm{kc} / \mathrm{s})\end{array}$ & $\begin{array}{c}\text { Output Count Rate } \\
(\mathrm{kc} / \mathrm{s})\end{array}$ & $\begin{array}{c}\text { FWHM (keV) } \\
\text { at 662 keV }\end{array}$ & $\begin{array}{c}\text { Processing Time } \\
(\text { seconds })\end{array}$ \\
\hline \multirow{2}{*}{$\begin{array}{c}\text { Trapezoidal } \\
\text { (rise time }=1.6 \mu \mathrm{s}, \\
\text { flat top time }=0.4 \mu \mathrm{s})\end{array}$} & 100 & 79.8 & 2.7 & 12 \\
\cline { 2 - 5 } & 200 & 141.0 & 3.9 & 13 \\
\cline { 2 - 5 } Template-matching & 100 & 96.6 & 2.8 & 160 \\
\cline { 2 - 5 } & 200 & 186.8 & 4.6 & 370 \\
\hline
\end{tabular}

\section{Conclusion}

To perform high-resolution spectroscopy measurements at an ultra-high throughput rate is a major challenge in active interrogation techniques for nuclear safeguards application. In this work, the development of advanced digital signal processing technique based on template-matching has been shown as an effective approach to address this issue. The principle behind the algorithm was described in detail. This algorithm was first tested using simulated piled-up pulses from a PMT coupled with a scintillator detector. Using a NaI detector, at moderate count rate $(\sim 200$ kcps), energy resolution comparable to traditional trapezoidal shaping was achieved ( $6.5 \%$ at $662 \mathrm{keV})$. With high rate data acquired with a silicon drift detector, the advantages of this new algorithm were demonstrated through comparison with the traditional approach using trapezoidal filters. Superior performance in terms of throughput rate and energy resolution was observed at high input rates. The performance of the template-match algorithm was then 
168

169

170

171

172

173

174

175

176

177

178

179

180

181

182

183

184

185

186

187

188

189

190

191

192

193

194

195

196

197

198

199

evaluated using signals from a HPGe detector. Despite the difficulty to construct an ideal template for pre-amplifier pulses, energy resolution of $4.6 \mathrm{keV}$ at $662 \mathrm{keV}$ and live time of $93.4 \%$ were successfully achieved at input rate of 200 kcps.

\section{Acknowledgement}

The authors would like to thank Dr. Paul Scoullar from Southern Innovation for providing the silicon drift detector data. This research is being performed using funding received from the DOE Office of Nuclear Energy's Nuclear Energy University Programs.

\section{References}

[1] R. Runkle, D. Chichester, S. Thompson, "Rattling nucleons: New developments in active interrogation of special nuclear material," Nucl. Instr. Meth. Phys. Res. A, vol. 663, pp 75 - 95, 2012.

[2] J. Dolan, M. Flaska, S. Pozzi, D. Chichester, "Passive measurementsofmixed-oxidefuelfornuclearnonproliferation," Nucl. Instr. Meth. Phys. Res. A, vol. 703, pp $102-108,2013$.

[3] B. Goddard, S. Croft, "High-fidelity passive neutron multiplicity measurements and simulations of uranium oxide," Nucl. Instr. Meth. Phys. Res. A, vol. 712, pp $147-156,2013$.

[4] E. Norman, et al., "Signatures of fissile materials: high-energy g rays following fission," Nucl. Instr. Meth. Phys. Res. A, vol. 521, pp 608 $610,2004$.

[5] R. Marrs, E. Norman, J. Burke, et al., " Fission-product gamma-ray line pairs sensitive to fissile material and neutron energy", Nucl. Instr. Meth. Phys. Res. A, vol. 592, pp 463 - 471, 2008.

[6] D. Beddingfield, F. Cecil, "Identification of fissile materials from fission product gamma-ray spectra," Nucl. Instr. Meth. Phys. Res. A, vol. 417, pp. 405-412, 1998.

[7] R. B. Walton, et al., "Delayed Gamma Rays from Photofission of U-238, U-235, and Th-232," Phys. Rev. B, vol. 134, pp. 824-832, 1964.

[8] S. Kahane, et al., "Photofission of U-238 with neutron-capture gamma rays," Phys. Rev. C, vol. 32, pp. 1944-1955, 1985.

[9] J. Jones, D. Norman, K. Haskell, et al., "Detection of shielded nuclear material in a cargo container," Nucl. Instr. Meth. Phys. Res. A, vol. 562, pp.1085-1088, 2006.

[10] X. Wen, J. Kavouras, D. Nakazawa, H. Yang, "Simulation and measurement of delayed $\gamma$-rays after photon-induced fission," Nucl. Instr. Meth. Phys. Res. A, vol. 729, pp 781-787, 2013.

[11] C. Hollas, D. Close, C. Moss, "Analysis of fissionable material using delayed gamma rays from photofission," Nucl. Instr. Meth. Phys. Res. $B$, vol. 24, pp. 503-505, 1987.

[12] M. Gmar, et al., "Assessment of actinide mass embedded in large concrete waste packages by photon interrogation and photofission," Appl. Radiat. Isot., vol. 63, pp. 613, 2005. 
200

201

202

203

204

205

206

207

208

209

210

211

212

213

214

215

216

217

[13] F. Carrel, M. Agelou, M. Gmar, et al., "Identification and differentiation of actinides inside nuclear waste packages by measurement of delayed gammas," IEEE Trans. Nucl. Sci., vol. 57, pp. 2862 - 2871, 2010.

[14] E. Reedy, S. Thompson, A. Hunt, "The detection of delayed gamma-rays between intense bremsstrahlung pulses for discriminating fissionable from non-fissionable materials," Nucl. Instr. Meth. Phys. Res. A, vol. 606, pp. 811-815, 2009.

[15] M. Kinlaw, A. Hunt, "Fissionable isotope identification using the time dependence of delayed neutron emission," Nucl. Instr. Meth. Phys. Res. A, vol. 562, pp. 1081-1084, 2006.

[16] T. Gozani, "Active Nondestructive Assay - Principles and Applications," NUREG/CR-0602, 1981.

[17] H. Yang, D. Wehe, D. Bartels, "Spectroscopy of high rate events during active interrogation," Nucl. Instr. Meth. Phys. Res. A, vol. 598, pp. 779-787, 2009.

[18] P. Scoullar, R. Evans, "Maximum likelihood estimation techniques for high rate, high throughput digital pulse processing," 2009.

[19] P. Scoullar, R. Evans, "Method and apparatus for resolving individual signals in detector output data," Patent No.: US 7,383,142 B2, 2008.

[20] M. Bolic, V. Drndarevic, W. Gueaieb, "Pileup correction algorithms for very-high-count-rate gamma-ray spectrometry with NaI(Tl) detectors," IEEE Trans. on Instrumentation and Measurement, vol. 59, 2010.

[21] B. Loher, " $\gamma$ - ray spectroscopy with a LaBr3:(Ce) scintillation detector at ultra-high count rates," M.S. thesis, 2010.

[22] V. Jordanov, G. Knoll, A. Huber, et al., "Digital techniques for real-time pulse shaping in radiation measurement," Nucl. Instr. Methods Phys. Res. A, vol. 353, pp. 261-264, 1994.

[23] T. Moon, "The expectation - maximization algorithm," IEEE signal processing magazine, November 1996.

[24] P. Scoullar, D. Scoullar, M. Brown, et al., "Advanced signal processing hardware for high throughput energy dispersive spectroscopy," NSLS Presentation, 2013. 


\section{igure 2}

Click here to download high resolution image

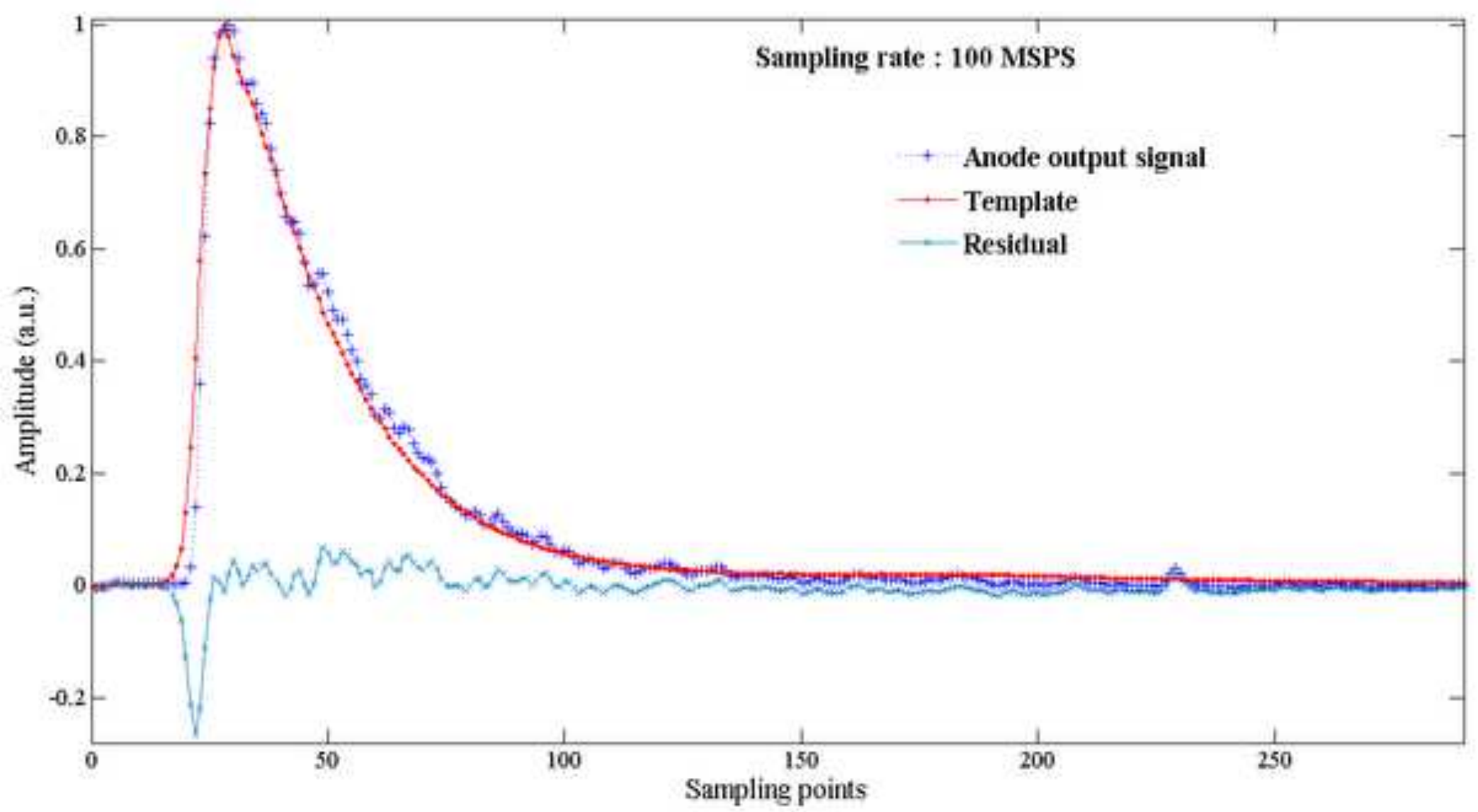




\section{Fure 7}

Click here to download high resolution image

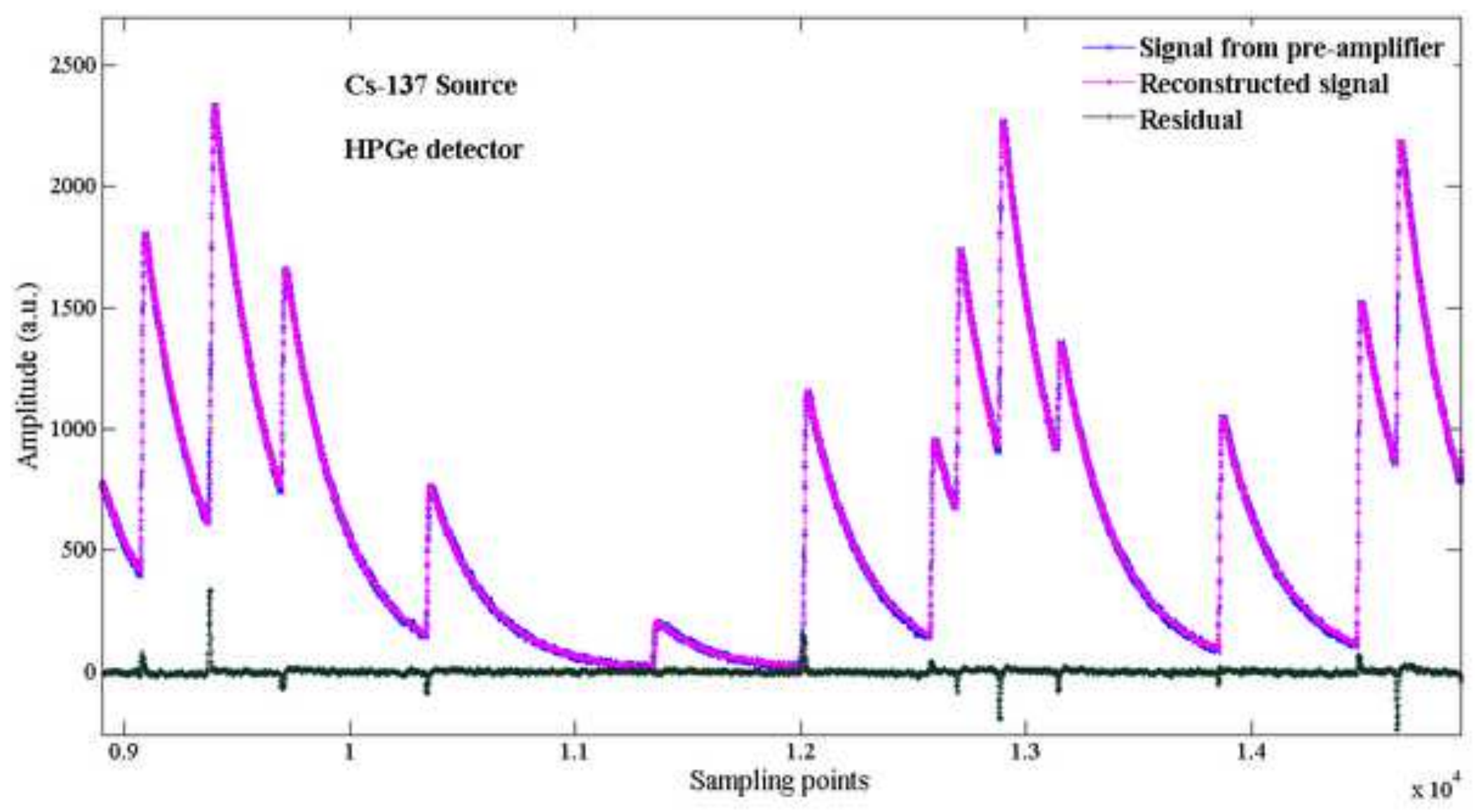

